\title{
Heart Wellness Programme: a pilot community-based cardiac rehabilitation programme in a multiethnic society
}

\author{
Yu Heng $\underline{K w a n}^{1,2,3}$, BSc, Kheng Yong $\underline{O n g}^{4}$, BSc, Hung Yong $\underline{T a y^{5}}$, BHSc, Joanne Yeh $\underline{\text { Chang }}{ }^{1}$, PharmD
}

INTRODUCTION Community-based exercise programmes have been shown to be effective in the Western world. However, there is a dearth of literature on their effectiveness in Asia. This pilot study aims to assess the effectiveness of the Asian community-based Health Wellness Programme (HWP) in Singapore. The HWP provides community cardiac rehabilitation services. METHODS A retrospective database analysis was conducted on cardiac rehabilitation patients who had completed the one-year wellness programme from 2010 to 2011. Patients were included in the programme if they had an underlying cardiac disease such as ischaemic heart disease and were deemed as stable by their cardiologist. Patients with New York Heart Association Class III or IV heart failure were excluded from this programme. Sociodemographic, anthropometric, clinical and laboratory data was recorded. Changes between the baseline and final measurements were analysed.

RESULTS Complete data from a total of 136 patients was analysed. Improvements were noted in body fat percentage (change $[\Delta]-1.3 \%, p<0.01)$, distance walked $(\Delta 9.7 \mathrm{~m}, \mathrm{p}=0.01)$, total cholesterol $(\Delta-7.8 \mathrm{mg} / \mathrm{dL}, \mathrm{p}=0.03)$, low-density lipoprotein $(\Delta-7.8 \mathrm{mg} / \mathrm{dL}, \mathrm{p}=0.03)$ and triglyceride $(\Delta-17.8 \mathrm{mg} / \mathrm{dL}, \mathrm{p}<0.01)$.

CONCLUSION The low-to-moderate intensity HWP seems to be effective in a multiethnic community setting. To confirm the quality and effectiveness of such a programme in secondary cardiovascular prevention, more evidence from prospective controlled trials with suitable controls is needed.

Keywords: Asia, community, exercise, rehabilitation

\section{INTRODUCTION}

Cardiovascular disease causes one-third of deaths worldwide, with 7.22 million deaths from coronary heart disease alone. ${ }^{(1}$ Asian countries host more than half of the world's population and have higher morbidity and mortality rates from stroke and coronary heart disease than their Western counterparts. ${ }^{(2)}$

Exercise has been found to be one of the best strategies to reduce the risk of coronary heart disease, ${ }^{(3)}$ while cardiac rehabilitation or secondary prevention is a core management strategy for patients with cardiovascular disease. ${ }^{(4)}$ The European Society of Cardiology defines cardiac rehabilitation as the sum of the interventions required to ensure the best possible physical, psychological and social conditions so that patients with chronic or post-acute cardiac disease may, through their own effort, preserve or resume their place in society. ${ }^{(5)}$

The Heart Wellness Programme (HWP), set up by the Singapore Heart Foundation, is an Asian community-based cardiac rehabilitation programme aimed at reducing cardiovascular events. Community-based exercise programmes in the West were shown to improve lipid panels, enhance quality of life, improve control of blood pressure and blood glucose, and reduce body weight, waist circumference and mortality. ${ }^{(6-8)}$ Although the HWP has been in operation since 2006, no studies have been done to validate its effectiveness in the community. Furthermore, to the best of our knowledge, no data has been published on the effectiveness of such a programme in Asian populations. Reported data is only available for countries such as Australia, the United Kingdom and the United States, and thus may not be applicable to Singapore, as the diet, environment and genetic make-up of the Asian population differs from that of the West. ${ }^{(9)}$

Therefore, this pilot study aimed to assess the effectiveness of the HWP in our local population. Our pilot protocol closely followed core component guidelines by the American Heart Association/American Association of Cardiovascular and Pulmonary Rehabilitation, consisting of management of risk factors (hypertension, diabetes mellitus, lipids, psychosocial factors and weight), patient assessment, nutritional counselling, smoking cessation, physical activity counselling and exercise training. ${ }^{(4)}$ With the preliminary information gathered, a prospective controlled trial can be planned to confirm our findings and, if the programme proves to be effective, public health workers can assess the feasibility of its expansion. The findings can also be used as a reference for health administrators in other Asian countries to start similar programmes in their communities to better manage cardiac rehabilitation and prevent cardiovascular disease progression.

\section{METHODS}

A retrospective electronic medical database analysis of the HWP was carried out on cardiac rehabilitation patients who had completed the one-year programme at the Singapore Heart Foundation from 1 January 2010 to 1 January 2011. Details of the patients' complete physical examinations, social demographics and clinical variables were collected at baseline and one-year follow-up. Relevant variables were race, gender, body weight,

${ }^{1}$ Department of Pharmacy, Faculty of Science, National University of Singapore, ${ }^{2}$ Program in Health Services and Systems Research, Duke-NUS Graduate Medical School, ${ }^{3}$ Department of Pharmacy, Khoo Teck Puat Hospital, ${ }^{4}$ Department of Pharmacy, Singapore General Hospital, ${ }^{5}$ Heart Wellness Centre, SHF-Isetan Foundation, Singapore Correspondence: Mr Kwan Yu Heng, MD-PhD Candidate, Program in Health Services and Systems Research, Duke-NUS Medical School, 8 College Road, Singapore 169857. yuheng@u.duke.nus.edu 
height, body and visceral fat, abdominal circumference, resting heart rate and blood pressure, body mass index (BMI), fasting lipid panel, six-minute walk distance, and smoking and past medical history (e.g. hypertension, dyslipidaemia, diabetes mellitus and overweight status). According to the World Health Organization's expert panel consultation on Asian BMI, a patient is classified as overweight if his/her BMI is $>23.0 \mathrm{~kg} / \mathrm{m}^{2}{ }^{2}{ }^{(10)}$ Institutional board review approval was obtained from the National University of Singapore and a waiver of patient consent was obtained.

Participants eligible for cardiac rehabilitation were those who had an underlying cardiac disease such as ischaemic heart disease and were clinically stable, as deemed by their cardiologist. As this was a community-based programme, patients with New York Heart Association Class III or IV heart failure were excluded from the HWP.

Prior to enrolment, programme staff recorded participants' complete medical and medication history, relevant laboratory parameters, and clinical data. Body weight as well as body and visceral fat were measured using a Tanita Body Composition Analyzer BC-418 (Tanita Corporation, Itabashi-ku, Tokyo, Japan). Measurements of visceral fat were strictly presented as a range; a range of $13-59 \mathrm{mg} / \mathrm{dL}$ implied that lifestyle modification was required to reduce the excess visceral fat to a range of $1-12 \mathrm{mg} / \mathrm{dL}$. A six-minute walk test was also conducted using American Thoracic Society guidelines. ${ }^{(11)}$ Participants were oriented on safety precautions before starting the exercise sessions.

During this programme, participants were required to undergo a series of exercise, nutritional, smoking cessation and psychosocial counselling sessions. All participants were required to maintain an exercise log. Programme staff also organised monthly support groups and ensured that health and safety guidelines were followed. Participants were required to attend a minimum of three exercise therapy sessions per week, at a cost of SGD 3 (about USD 2.41) per session. Each 1.5-hour session involved a combination of warm-up, aerobics, resistance training and cool-down exercises. The exercise programme was at a low-to-moderate intensity with a maximum oxygen consumption of $60 \%-80 \%$; this varied according to the individual's physical condition. Participants were required to have their physical and clinical variables remeasured with relevant laboratory parameters provided by their physicians.

All statistical analyses were carried out using STATA SE version 12.1 for Windows (StataCorp LP, College Station, TX, USA). Continuous variables were expressed as mean \pm standard deviation and categorical variables as number (percentage). Differences in comparisons over a year were analysed using either paired $t$-test or Wilcoxon signed rank test for normal and nonnormal distributed continuous data, respectively. A p-value $<0.05$ was considered statistically significant.

\section{RESULTS}

A total of 136 patients (mean age $65.9 \pm 8.7$ years) were included in our analysis, of whom 116 (85\%) were men. Baseline characteristics of the recruited patients are presented in Table I.

Table Il shows the patient measurements at one-year follow-up. Patients experienced an overall decrease in body fat percentage (change $[\Delta]-1.3 \%$, 95\% confidence interval $[\mathrm{Cl}]-1.9 \%$ to $-0.9 \%$ )
Table I. Baseline characteristics of patients $(n=136)$.

\begin{tabular}{lc}
\hline Characteristic & No. (\%) \\
\hline Age* (yr) & $65.9 \pm 8.7$ \\
Gender & \\
Male & $116(85)$ \\
Female & $20(15)$ \\
Height* (m) & $1.7 \pm 0.1$ \\
Race & \\
Chinese & $117(86)$ \\
Indian & $8(6)$ \\
Malay & $3(2)$ \\
Other & $8(6)$ \\
Diabetes mellitus ${ }^{+}$ & $40(29)$ \\
Hypertension ${ }^{+}$ & $90(66)$ \\
Dyslipidaemia ${ }^{+}$ & $109(80)$ \\
Overweight & $44(32)$ \\
History of smoking & $3(2)$ \\
Number of chronic diseases & $10(7)$ \\
0 & $33(24)$ \\
1 & $73(54)$ \\
2 & $20(15)$ \\
\hline
\end{tabular}

*Data presented as mean \pm standard deviation. ${ }^{+}$Patients with official diagnosis of conditions. \#Body mass index $>23.0 \mathrm{~kg} / \mathrm{m}^{2}$.

and increase in six-minute walk distance $(\Delta 9.7 \mathrm{~m}, 95 \% \mathrm{Cl} 2.0 \mathrm{~m}$ to $17.5 \mathrm{~m})$, abdominal circumference $(\Delta 1.0 \mathrm{~cm}, 95 \% \mathrm{Cl} 0.3 \mathrm{~cm}$ to $1.6 \mathrm{~cm})$ and visceral fat density $(\Delta 0.5 \mathrm{mg} / \mathrm{dL}, 95 \% \mathrm{Cl} 0.3 \mathrm{mg} / \mathrm{dL}$ to $0.9 \mathrm{mg} / \mathrm{dL}$ ).

Only 43 patients had a complete fasting lipid profile; they experienced a decrease in total cholesterol $(\Delta-7.8 \mathrm{mg} / \mathrm{dL}$, $95 \% \mathrm{Cl}-15.6 \mathrm{mg} / \mathrm{dL}$ to $-3.9 \mathrm{mg} / \mathrm{dL}$ ), low-density lipoprotein $(\Delta-7.8 \mathrm{mg} / \mathrm{dL}, 95 \% \mathrm{Cl}-15.6 \mathrm{mg} / \mathrm{dL}$ to $-3.9 \mathrm{mg} / \mathrm{dL})$, and triglyceride levels ( $\Delta-17.8 \mathrm{mg} / \mathrm{dL}, 95 \% \mathrm{Cl}-26.7$ to $-8.9 \mathrm{mg} / \mathrm{dL}$ ).

\section{DISCUSSION}

To the best of our knowledge, this is the first Asian study performed on a community-based cardiac rehabilitation programme aimed at reducing the progression of cardiovascular disease. Overall, patients that underwent one-year follow-up with the HWP experienced a reduction in body fat percentage, were able to walk a longer distance, and improved total cholesterol and lowdensity lipoprotein control. These findings are comparable to that of an Australian study done by Richardson et al. ${ }^{(8)}$ Our results are also similar to that of a controlled trial by Bethell et al that showed similar benefits in weight and lipid control. ${ }^{(12)}$ Although our study saw only a modest improvement of $9.7 \mathrm{~m}$ in the sixminute walk test, the majority of our patients achieved a final walking distance of $547.6 \mathrm{~m}$, which was better than the findings (514.21 m) of a recently published trial on cardiac rehabilitation patients by Ghashghaei et al. ${ }^{(13)}$ Compared to the aforementioned trial, our patients also showed similar or better end-point results in terms of diastolic blood pressure. ${ }^{(13)}$ Moreover, our patients were healthier and had better blood pressure profiles compared to those of other published studies. ${ }^{(12,13)}$ 
Table II. Comparison of changes in patients' measurements.

\begin{tabular}{|c|c|c|c|c|}
\hline Measurement & Baseline & Change & Final & p-value \\
\hline Weight (kg) & $67.0(65.0,68.9)$ & $-0.1(-0.6,0.4)$ & $66.9(65.0,68.8)$ & 0.68 \\
\hline Body mass index $\left(\mathrm{kg} / \mathrm{m}^{2}\right)$ & $24.4(23.8,24.9)$ & $0.0(-0.2,0.1)$ & $24.4(23.8,24.9)$ & 0.84 \\
\hline Abdominal circumference $(\mathrm{cm})$ & $89.4(87.9,90.9)$ & $1.0(0.3,1.6)$ & $90.4(88.9,91.8)$ & $<0.01$ \\
\hline Pulse (bpm) & $66.3(64.5,68.0)$ & $0.7(-0.7,2.1)$ & $67.0(65.4,68.7)$ & 0.32 \\
\hline SBP (mmHg) & $124.2(122.1,126.3)$ & $0.6(-3.2,2.1)$ & $123.6(121.2,126.1)$ & 0.69 \\
\hline Body fat (\%) & $26.4(25.4,27.5)$ & $1.3(-1.9,-0.9)$ & $25.1(23.9,26.2)$ & $<0.01$ \\
\hline Visceral fat density (mg/dL) & $12.4(11.6,13.1)$ & $0.5(0.3,0.9)$ & $12.9(12.3,13.6)$ & $<0.01$ \\
\hline 6-min walk (m) & $537.9(523.9,551.9)$ & $9.7(2.0,17.5)$ & $547.6(532.4,562.9)$ & 0.01 \\
\hline \multicolumn{5}{|l|}{ Lab variables $(n=43)$} \\
\hline $\mathrm{TC}(\mathrm{mg} / \mathrm{dL})$ & $156.0(148.2,167.7)$ & $-7.8(-15.6,-3.9)$ & $148.2(140.4,156.0)$ & 0.03 \\
\hline $\mathrm{HDL}(\mathrm{mg} / \mathrm{dL})$ & $46.8(42.9,50.7)$ & $3.9(-3.9,3.9)$ & $50.7(46.8,54.6)$ & 0.16 \\
\hline $\mathrm{LDL}(\mathrm{mg} / \mathrm{dL})$ & $89.7(81.9,97.5)$ & $-7.8(-15.6,-3.9)$ & $81.9(78.0,89.7)$ & 0.03 \\
\hline $\mathrm{TG}(\mathrm{mg} / \mathrm{dL})$ & $97.9(80.1,106.8)$ & $-17.8(-26.7,-8.9)$ & $80.1(71.2,89.0)$ & $<0.01$ \\
\hline
\end{tabular}

Data presented as mean (lower limit, upper limit). bpm: beats per minute; DBP: diastolic blood pressure; HDL: high-density lipoprotein; LDL: low-density lipoprotein; SBP: systolic blood pressure; TC: total cholesterol; TG: triglyceride

We found increased abdominal circumference and visceral fat in our patients, despite improvements in body fat percentage. This finding is novel compared to that of other similar studies performed on the Western population. ${ }^{(6,8)}$ One possible reason for this finding is that the environmental influences and genetic make-up of Asians, compared to Westerners, resulted in increased abdominal obesity regardless of changes in body fat. ${ }^{(9)}$ The high carbohydrate intake, particularly starch, in Asian diets compared to those of Westerners could also be a contributing factor. ${ }^{(14)}$ The findings from our study did not point to the failure of the HWP; on the contrary, they showed the programme's potential in delaying disease progression and preventing further complications, despite the low exercise intensity.

There were several limitations to our study. This was a pilot study without appropriate controls; thus, cause-and-effect relationships could not be established. Laboratory measurements were also incomplete, as data on lipids and glycated haemoglobin was not measured in all patients. This limited our understanding of the programme's effectiveness in chronic diseases such as hyperlipidaemia and diabetes mellitus. Due to the cross-sectional nature and short duration of this study, patients, enrolling at varying times, only had data collected during the selected oneyear period. This may have resulted in a less effective outcome. Furthermore, as this was a retrospective study, important data such as the intensity of the exercise sessions was not recorded. A prospective controlled trial with suitable controls and complete laboratory parameters is currently in progress to overcome these limitations. Until then, we hope to establish a more holistic view of secondary cardiac prevention in the community setting.

In conclusion, with the increasing prevalence of cardiovascular disease in Asia, a community-based HWP is important to reduce cardiovascular risk in Asian populations. We have shown that the HWP has been associated with favourable benefits such as improved walking distance and fasting lipid profile, and a reduction in body fat. Understanding the effectiveness of the HWP can provide evidence for its possible expansion, widening its impact on society.

\section{ACKNOWLEDGMENT}

We thank the Singapore Heart Foundation for the provision of the data.

\section{REFERENCES}

1. Heran BS, Chen JM, Ebrahim S, et al. Exercise-based cardiac rehabilitation for coronary heart disease. Cochrane Database Syst Rev 2011; (7):CD001800.

2. Ueshima H, Sekikawa A, Miura K, et al. Cardiovascular disease and risk factors in Asia: a selected review. Circulation 2008; 118:2702-9.

3. Tanasescu M, Leitzmann MF, Rimm EB, et al. Exercise type and intensity in relation to coronary heart disease in men. JAMA 2002; 288:1994-2000.

4. Balady GJ, Ades PA, Comoss P, et al. Core components of cardiac rehabilitation/secondary prevention programs: A statement for healthcare professionals from the American Heart Association and the American Association of Cardiovascular and Pulmonary Rehabilitation Writing Group. Circulation 2000; 102:1069-73.

5. Gohlke H, Gohlke-Bärwolf C. Cardiac rehabilitation. Eur Heart J 1998; 19:1004-10.

6. Minges KE, Cormick G, Unglik E, Dunstan DW. Evaluation of a resistance training program for adults with or at risk of developing diabetes: an effectiveness study in a community setting. Int J Behav Nutr Phys Act 2011; 8:50.

7. Miller TD, Balady GJ, Fletcher GF. Exercise and its role in the prevention and rehabilitation of cardiovascular disease. Ann Behav Med 1997; 19:220-9.

8. Richardson G, van Woerden HC, Morgan L, et al. Healthy hearts--a community-based primary prevention programme to reduce coronary heart disease. BMC Cardiovasc Disord 2008; 8:18.

9. Elder SJ, Lichtenstein AH, Pittas AG, et al. Genetic and environmental influences on factors associated with cardiovascular disease and the metabolic syndrome. J Lipid Res 2009; 50:1917-26.

10. Choo V. WHO reassesses appropriate body-mass index for Asian populations. Lancet 2002; 360:235.

11. ATS Committee on Proficiency Standards for Clinical Pulmonary Function Laboratories. ATS statement: guidelines for the six-minute walk test. Am J Respir Crit Care Med 2002; 166:111-7.

12. Bethell HJ, Mullee MA. A controlled trial of community based coronary rehabilitation. Br Heart J 1990; 64:370-5

13. Ghashghaei FE, Sadeghi M, Marandi SM, Ghashghaei SE. Exercise-based cardiac rehabilitation improves hemodynamic responses after coronary artery bypass graft surgery. ARYA Atheroscler 2012; 7:151-6.

14. Zhou BF, Stamler J, Dennis B, et al; INTERMAP Research Group. Nutrient intakes of middle-aged men and women in China, Japan, United Kingdom, and United States in the late 1990s: the INTERMAP study. J Hum Hypertens 2003; 17:623-30. 